\title{
Using the Photovoice Method to Elicit Authentic Learning in Online Discussions
}

\author{
Beatriz Reyes-Foster \\ University of Central Florida \\ Beatriz.reyes-foster@ucf.edu \\ Aimee deNoyelles \\ University of Central Florida \\ aimee.denoyelles@ucf.edu
}

\begin{abstract}
While online discussions remain popular in college classrooms, mixed results persist about their effectiveness in eliciting authentic learning. This case study explores how students perceive the influence of the Photovoice method on their authentic learning, critical thinking, engagement, and peer interaction in an asynchronous online discussion. Photovoice is a research method combining photography with social action, in which people express their points of view by photographing scenes that highlight certain themes. Students in an online undergraduate course engaged in an online discussion which asked them to connect personal images to the course content. Students reported that this strategy supported authentic learning, critical thinking, engagement, and interaction; in addition, a correlational analysis found that these factors are highly interrelated. This case study proposes recommendations for practitioners interested in using a similar approach.
\end{abstract}

Keywords: Photovoice, authentic learning, active learning, online discussions, online learning and teaching, undergraduate education.

\section{Introduction}

The growth of online learning in college courses over the last decades has provided students with new opportunities to complete a college degree with a plethora of flexible learning options, making a college education more accessible than ever before. Alongside the shift towards online learning has been an increase in ownership of mobile technologies (Dahlstrom \& Bichsel, 2014), particularly technologies with photographic capabilities (Chen, Seilhamer, Bennett, \& Bauer, 2015). With the rise of online photo-sharing services like Instagram and the incorporation of photo-sharing into popular social networking platforms like Facebook and Twitter, Millennials have come to earn the moniker of “The Self(ie) Generation” (Blow, 2014).

Integrating this personal practice into existing classroom practices may set the stage for active learning to take place. At its simplest definition, students actively learn when they are “doing things and thinking about what they are doing” (Bonwell \& Eison, 1991, p. 3). A more nuanced conception of the term includes higher-order thinking tasks such as analyzing and reflecting, as well as creating meaningful connections to prior knowledge and applying new knowledge to different contexts (Fiorella \& Mayer, 2015). Active learning serves as an umbrella concept for other subsets of learning, such as authentic learning. The core goals of authentic learning are to enable students to "learn by doing": thinking and solving problems like a 
professional, constructing concepts and relationships in contexts that involve real-world settings, and creating a product to be shared with the community (Lombardi, 2007).

Authentic learning is a challenging approach in face-to-face teaching; however, it presents unique challenges in an online environment. Instructors who wish to foster authentic learning in an online setting must identify effective tools to facilitate it. Mobile devices that enable photographic sharing show promise, but in order for students to meaningfully learn, the devices must be utilized in meaningful ways. Another tool that has this potential in an online course is the asynchronous discussion forum. Once purely text-based, most online discussion forums now allow for media uploads such as images and videos, therefore allowing students to capture media with their mobile devices and then share with others through the discussion forum.

To promote active learning in a classroom setting, discussion is acknowledged as one of the most commonly used strategies; however, it must foster a supportive environment that encourages risk taking (Bonwell \& Eison, 1991). Students often do not see the value in actively participating in online discussions and do the bare minimum to complete the requirements (Hew, Cheung, \& Ng, 2010). Research on the effectiveness of online discussions has yielded mixed results, with most research concluding that the design of the prompt and the way the discussion is facilitated play significant roles in whether or not the strategy will be successful (deNoyelles, Zydney, \& Chen, 2014; Mazzolini \& Madison, 2007).

An online discussion that supports authentic learning will prompt students to ask questions, exchange, connect, and expand on ideas, as well as apply new ideas to other contexts. Media elements, such as image sharing, can facilitate this process. Specifically, strategies that support students to be socially and cognitively present are necessary. deNoyelles et al. (2014) found that discussions which incorporated a peer facilitation element and discussions which were explicitly structured to support student dialogue were especially effective in supporting an online community; not only the students' learning, but also the ease of the teachers' facilitation. In addition, incorporating media elements such as audio-based feedback was found to increase the level of teaching presence perceived by students.

In this case study, the researchers sought to harness the power of mobile technologies, particularly phone cameras, to enable student-created media and to introduce a teaching strategy that would incorporate Photovoice, a research methodology with origins in social action research, to promote authentic learning in online discussions. The researchers formulated the following research question: How do students perceive the influence of the Photovoice method on their authentic learning, critical thinking, engagement, and peer interaction in an asynchronous online discussion?

\section{Photovoice}

The roots of Photovoice lie in social action and justice research, and the method was originally conducted in face-to-face environments. The purpose of Photovoice is for the participants to take pictures in the community that represent something meaningful to them, and then peers in the community interpret them. The final product results in a collage of the participants' pictures, which are critically interpreted by the whole community (Kroeger \& Meyer, 2005). Implications and actions are then generated from the collage. This method can be applied to the online discussion realm in order for students to connect what they are learning in the classroom to their prior knowledge and real-world contexts, which will be naturally diverse and unique. Photovoice is a particularly appropriate choice for fostering authentic learning in an anthropology course in 
particular because the method is frequently used by anthropologists (Johnson, 2011). Thus, not only are the students actively applying course concepts through the lens of their daily lives, they are also learning to apply a research method used in anthropology.

The Photovoice method has been employed in varied educational settings, but rarely in online discussions. In a literature search using the key terms "photovoice and online discussion," the few studies that were identified typically focused on using photovoice as a research method, rather than a classroom-based intervention (see Jecklin, 2010; LaDonna \& Ravenek, 2014). Of the few that utilized photovoice as a classroom-based intervention, most were in face-to-face settings (see Bonnycastle \& Bonnycastle, 2015; Cook \& Quigley, 2013; Schell, Ferguson, Hamoline, Shea, \& Thomas-Maclean, 2009) or used a digital aspect but not specifically in an online discussion forum (see Edwards, Perry, Janzen, \& Menzies, 2012; Gubrium \& Torres, 2013). The findings shared in this case study add to the research body by considering the implementation of the method into online discussions.

\section{Methods}

\section{Participants and Context}

Research participants ( $\mathrm{N}=67)$ were recruited from one online section (enrollment 135) of an undergraduate anthropology course at a large public university in the southeastern United States in the spring semester of 2016. All participants were students enrolled in Language and Culture, a required upper-level anthropology course that satisfies requirements in several other majors and certificate programs. Of the entire course, the largest proportion of students (38\%) were anthropology majors, followed by Library Sciences (12\%), and Psychology, Elementary Education, Computer Science, and Political Science (4\% each). Other disciplines represented were Visual Arts, Biomedical Sciences, Health Sciences, English, Math, Spanish, and Philosophy. In terms of class standing, $61 \%$ of the students were Seniors, $33 \%$ of the students were Juniors, $4 \%$ were Sophomores, and $2 \%$ were second-degree seeking students. We did not collect data on age or gender identity.

The course was held entirely online and did not have any face-to-face meeting requirements. Author 1 was the instructor of record for this class. Author 2 was in charge of student recruitment into the study, which was conducted through the dissemination of an announcement using the university Learning Management System. Students were given an option to opt in to the study and received an incentive of 3\% extra credit added to their final grade. Students who did not opt into the study were given the opportunity to complete an alternative assignment for the same extra credit. Students who opted in to the study did not have to do anything other than the assignment requirements, specifically complete the discussion and answer a feedback survey. The instructor did not know who participated in the study until after the online discussion had been graded.

In this course, online graded discussions were worth $40 \%$ of the final grade. Students also had a midterm and final exam requirement (20\%), five online content-based quizzes (15\%), and two short papers, one a reflexive language autobiography, the other a research-based profile of an endangered language (25\%). The discussion strategy analyzed was the fourth graded discussion of the term, submitted after the mid-term exam. The graded discussion was designed to evaluate student knowledge of the concepts of "performance" and "performativity," which were covered as part of the regular course content (Ahearn, 2012). It was worth approximately 
$8 \%$ of the final grade. All students in the class were divided into 15 groups of 8-10 students, which remained constant throughout the semester. The purpose of the discussion design was to connect course materials -specifically, the linguistic anthropological concept of "performance"-into the students' real lives, thereby allowing them to "think like anthropologists.”

\section{Discussion Prompt}

All students were required to complete the following prompt:

'Photovoice' is a research method in which people express their points of view or communities by photographing scenes that highlight certain themes - then the photographs are interpreted in the community. At the end, we have this nice collection of photos that represent certain themes. This week, we have spend a lot of time talking about performance and performativity. For this discussion, I want you to think outside the box.

Using your cell phone or camera, take a picture of a performance --remember, our definition of "performance" is very broad, so this can be a display of identity, a speech, or a particular event. YOU MUST TAKE THE PICTURE YOURSELF, images from the internet will not be accepted.

There are three ways to upload the image to the discussion board.

1. One way is to use the Canvas mobile app to upload your picture to this discussion (for details about downloading the app, visit http://online.ucf.edu/support/webcourses/other/ucfmobile/).

2. You can also upload the picture using your computer; to do that, within the discussion post, click on the 'Embed Image' icon (next to the pi sign) in the editing toolbar, then click on the Canvas tab, and then find your image in My Files (note: you must have uploaded the image to your My Files (Links to an external site.) area first).

3. Finally, you can attach your image file to the discussion post.

\section{FOR TUESDAY:}

1. Post the picture to the discussion board. Do NOT include an interpretation of the picture.

2. Choose one of your fellow group member's pictures and write a 100-200 word response, interpreting the image. What is the image of? Why would it be considered a performance? How do the concepts of performance, performativity (including the performativity of gender), and performance as display of verbal artistry help us interpret this image?

For THURSDAY:

1. Respond to the reaction posted on your photo in less than 100 words and reveal the concept you were trying to illustrate. Reflect on whether the person was on target or what you saw that was different.

2. Respond to the feedback survey. 
As displayed in the discussion prompt, the researchers ensured that the purpose of Photovoice was well articulated and technical instructions for uploading images to the discussion board were provided. In addition, due date reminders were created within the learning management system so that students would be reminded of the multiple parts of the discussion.

\section{Discussion Artifacts}

An example of an image provided by Student 1 is in Figure 1. Student 2 interpreted this photo in Part B of the online discussion: "I love your example of a performance I considered using a photo from the parade myself! This is a very easy example of a performance. Someone inside the suit is taking on the role of the character and performing as them. Another fun interesting thing about the performance is you can tell the character while a duck is supposed to be a female duck. The way the clothes are used, the bow, and the elongated eyelashes. By the way the hands are being held (and from seeing this parade myself) I can tell that it's mid motion of greeting or dance along with the music that is normally played. A performance within a performance.”

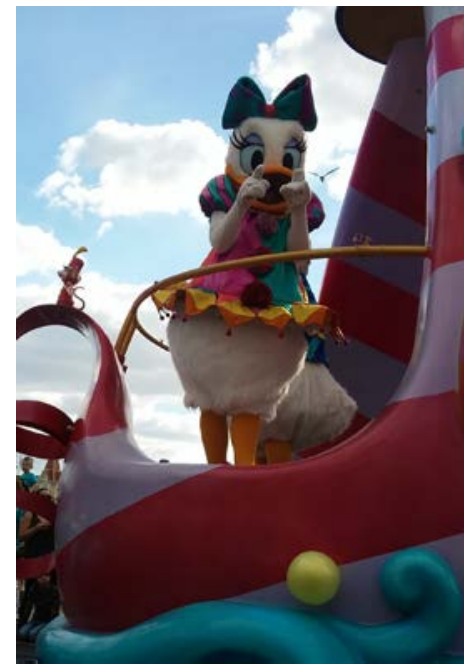

Figure 1. Example of Part A discussion response.

In Part C, Student 1, who took the picture in Figure 1, reflected on Student 2's interpretation; "I thought to use a photo from the parade because it has performers conveying a variety of characters with different associated identities, relying on the competence of the audience. In this photo, the actor is performing Daisy Duck, actively constructing what that character means to the audience while playing on their existing ideas of what she is like, and performing a gender. You pointed these aspects out right away. You are right about her greeting -- I don't know if you can tell from the still photo, but she is blowing me a kiss. Notably, the illocutionary action of blowing a kiss, which could be considered performatively equivalent to kissing someone, has more specific flirtatious intention than some other affectionate acts in our culture. A performer representing Daisy years ago when the park opened might not have been willing to blow a kiss to a female fan as part of her characterization.” 


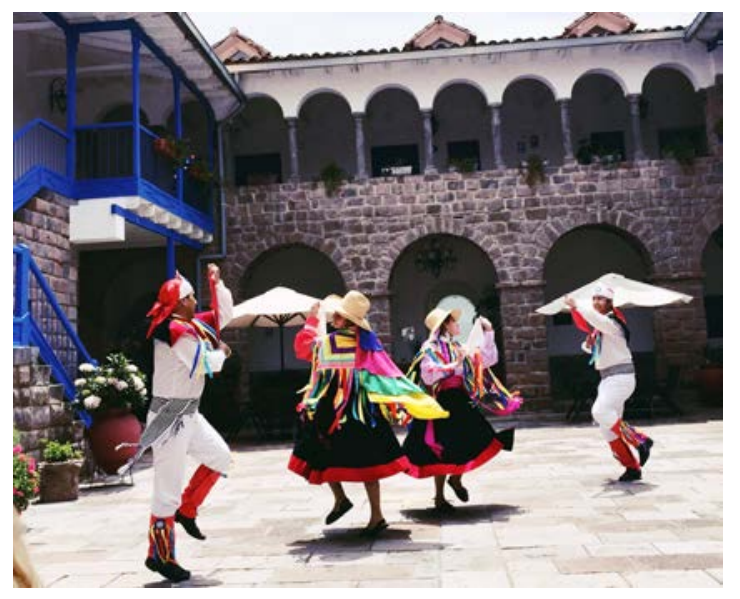

Figure 2. Example of Part B discussion response.

Student 3 provided the image in Figure 2. Student 4 analyzed this image in the following way: "In this image, men and women are dancing together in pairs in colorful costumes. Their dance and appearances represent a variety of different concepts they are performing to an audience with the competence to understand them. For one thing, they are using dancing to perform an idea. I do not know the context of why they are dancing, but from their bright costumes, they may be performing a celebration. I think this because I am operating on the cultural assumption that bright colors represent something positive, which I assume is part of the 'competence' (Ahearn 2012, 161) the dancers have. The performativity of gender (Ahearn 2012, 162) also plays a role here. The men and women wear distinct costumes to differentiate them so they can perform their genders distinctly, and they dance in pairs based on those genders. If I could hear them, I could determine whether they are displaying verbal artistry, but their performance appears to be mostly based around nonverbal communication."

In Part C, Student 3 responds; "You are correct in your interpretation of performativity of gender as well as the use bright colors and non verbal expression. I took this photo in Cusco, Peru while I was observing tradition Quechuan dance. This particular dance was a performance of identity - an expression of self and cultural values manifested through movement. What the picture does not capture is the narrative-style song that the dancers are interpreting. Quechuan culture does not have an extensive history of orthography [sic] so much of Quechuan history is portrayed through stories, ceremonies, weavings, or song-and-dance performances such as this."

\section{Data Collection and Analysis}

This study took a mixed-methods approach, in that both quantitative and qualitative data was collected for analysis. After completing the discussion activity, all research participants completed a survey (Appendix A) containing 30 closed-ended items (5-point Likert scale) and one open-ended item that aimed to measure their perceptions of authentic learning, critical thinking and engagement, as well as peer interaction. Survey items were generated from the various definitions and coding schemes employed in previous articles (see Behar-Horenstein \& Niu, 2011; Yang, Newby, \& Bill, 2005). Cronbach’s alpha based on standardized items for the authentic learning scale was .83, critical thinking scale was .91 peer interaction was .80, and engagement was .90 , which are all acceptable levels for reliability. Descriptive statistics were used, and correlations between the factors were measured. The open-ended item, which simply 
asked students to provide feedback about the discussion, was analyzed for emerging themes related to authentic learning, critical thinking and engagement and used to complement and expand understanding of the quantitative results.

\section{Results}

\section{Correlations}

Correlations between authentic learning, critical thinking, engagement, and peer interaction were calculated. Relationships between factors were all significant at the 0.01 level (Table 1 ).

Table 1. Correlations.

\begin{tabular}{|l|l|l|l|l|}
\hline & $\begin{array}{l}\text { Critical } \\
\text { Thinking }\end{array}$ & Engagement & $\begin{array}{l}\text { Authentic } \\
\text { Learning }\end{array}$ & Engagement \\
\hline $\begin{array}{l}\text { Critical } \\
\text { Thinking }\end{array}$ & & .78 & .83 & .83 \\
\hline Engagement & .78 & & .73 & .77 \\
\hline $\begin{array}{l}\text { Authentic } \\
\text { Learning }\end{array}$ & .83 & .73 & & .80 \\
\hline Interaction & .83 & .77 & .80 & \\
\hline
\end{tabular}

It was found that the relationship between critical thinking and authentic learning was most significant $(\mathrm{r}=.83, \mathrm{p}<.01)$. Participants who reported higher levels of authentic learning were also very likely to report higher levels of critical thinking. However, when controlling for engagement and interaction, the correlation between authentic learning and critical thinking was noticeably weaker $(r=.44)$. This suggests that all factors indirectly contribute toward the experience. Student comments below expand on this finding.

\section{Authentic Learning and Critical Thinking}

Concerning authentic learning, most students agreed that the discussion made them put their knowledge of "performance" into action $(\mathrm{M}=4.73)$ and prompted them to relate their experience of the world to the course content $(\mathrm{M}=4.52)$. One student shared, "I loved the photovoice assignment! It [the discussion prompt] encouraged me to view the world through the lens of the topic I'm discussing." This statement precisely coincides with the discussion goal: to apply the course concept to "real life." Students agreed that only being provided with an image encouraged them to think more critically yet openly about the term "performance" $(\mathrm{M}=4.70)$. One student explained, "Instead of being told what to think about a picture or informed what was going on ahead of time, this assignment allowed for thinking outside the box and use of life experiences to interpret the photo." This comment suggests that the discussion structure encouraged the risktaking that is necessary for true active learning to take place. Interpreting others' images prompted students to think about the concept of "performance" in multiple ways $(\mathrm{M}=4.70)$. In their comments, students frequently spoke about the discussion making them "think outside the 
box" and "use... life experiences," supporting the finding that the discussion fosters a unique mix of higher-order critical thinking skills and authentic learning.

The overlap of authentic learning with critical thinking was a recurrent theme. For instance, as one student explained, "Before I chose a picture, I had to analyze the things I typically do and encounter, which helped my understanding of the material." This statement suggests that the student had to reflect on how the assigned concept manifested in their day-today life, in order to capture an image that represented the concept. Another shared, "I really enjoyed this assignment, because I had to look for a photo and it really made me think more about the assignment and choosing a photo that represented what I wanted to portray." This comment exemplifies the active element of the learning; the student was not only doing things, but thinking about what they were doing. The discussion prompted students to connect their prior knowledge and apply their real-life experiences to the course content in a meaningful way. One student noted, "The assignment allowed me to be more actively aware of the discussion because I had to think critically on photos that I have taken in the past compared to thinking of what would be considered a proper image for the assignment, I had to go back to the course content a few times to make sure I fully understood what was expected of me. Which made me understand the content better and I feel confident of my image choice and the discussion assignment as a whole." This statement displays how the reciprocal relationship between authentic learning and critical thinking was realized through the Photovoice method. Specifically, the statement above illustrates the way in which the discussion, by encouraging the student to connect the course material to their lived experiences, deepened the student's understanding of the course concepts.

\section{Engagement and Interaction}

Engagement in the discussion was high. Students agreed that the discussion was valuable $(\mathrm{M}=$ 4.63) and that they were more motivated to participate in this discussion when compared to previous discussions $(\mathrm{M}=4.51)$. Students agreed that interpreting the images that their peers had posted made them feel more engaged in the activity $(\mathrm{M}=4.55)$. A student explained, "I was excited to see what other students would post as their image because everyone can come up with something different in their everyday life." Another highlights how this extends to critical thinking: "It was interesting to discuss the images because...looking at them without context forces you to think [about] what they mean." In addition, students agreed that the discussion prompt resulted in an interesting product to be shared with the class community $(\mathrm{M}=4.27)-\mathrm{a}$ key element of the Photovoice method.

Lastly, the discussion promoted interactions with peers $(\mathrm{M}=4.60)$. One student noted, "This is a great assignment because it takes us away from the usual book work for a while and lets us interact in a more comfortable and casual way where we can express ourselves through an image." While characterizing the discussion as "comfortable" and "casual," the student notes that even though the discussion does ask students to connect the images to the course content, it simultaneously allows for creative expression, a welcome break from "book work." Analyzing the images taken within the groups encouraged students to reflect on their understanding of the course content $(\mathrm{M}=4.52)$. Reading peers' responses about the student's image encouraged them to reflect on their personal understanding of the course content, which is a core element of active learning $(\mathrm{M}=4.37)$.

The relationship between peer interaction and engagement is illuminated through student comments. One student shared, "I felt more connected to my peers. It was interesting seeing the 
different pictures people posted about various performances. I had a ton of fun doing this discussion." In an online class of nearly 150 students, genuine peer-to-peer connectedness is difficult to create, yet several students expressed themselves positively about newfound connections to their peers. For instance, another student said, "This discussion made me more connected with my students. It required me to respond to their responses on my photo and it made me more engaged and excited for the posts." Signaling a change from the norm, one student shared, "This was a fun discussion. I actually looked forward to any response I would receive from a classmate(s) and responding." Interestingly, the requirement of interaction did not dampen students' spirits; “I think this assignment was really interesting. I especially liked how we were required to respond to our peer's response to our photo." Drawing on affective language such as "ton of fun," and "excited," these student comments suggest that the engagement, interaction, and pleasure were closely connected.

\section{Visual Nature}

While we did not intend to directly study it, the visual nature of the discussion prompt invited powerful comments from students. First, photo sharing appears to promote a new connection among students not often realized in the online classroom. One student exclaimed, "The use of images made me feel more connected to the students and it made the discussion really fun." Second, introducing a medium other than text was appreciated. Another explained, "I enjoyed this discussion much more than other discussions because, for one, it was much more visual. As a visual learner, I was able to apply the concepts we learned to the photos I looked at." Third, varying the discussion elements can support not only a varying array of learning styles, but also those within disciplines. For example, one student shared, "I am not an anthropology major. Most of my coursework has been in art history and visual arts. So I found this type of assignment more interesting and easier than the regular discussion posts."

Finally, it also appears that the visual element subtly encouraged a level of risk-taking typically not found in text-based discussions. For example, when the student quoted above says, "It was interesting to discuss the images because...looking at them without context forces you to think [about] what they mean," this statement, while indicative of higher-order thinking and analysis, also suggests risk-taking, as they are "forced" to think in a different way. The visual nature of the discussion also encouraged risk-taking by compelling students to interact with one another, a sometimes arduous task in an online setting.

\section{Discussion}

Results of this exploratory study bear several implications. First, the results suggest that the Photovoice method has the potential to support students' active and authentic learning in an online discussion, as well as promote other elements such as critical thinking, engagement, and interaction. Several factors contribute to this; perhaps most importantly, the opportunity to creatively express personal connections to the course content in ways other than through the "traditional" text-based online discussion. When carefully crafted, students can make sense of the course content with others in a way that is relevant to them, using a medium that is second nature in their personal lives.

The study findings also highlight the importance of varying up the elements of online discussions, especially if discussions are used extensively in a course. Discussion forums now 
often support multiple media elements, including images and videos, presenting an opportunity to take advantage of those elements. Discussing images appeared to engage students more in the activity, enhance interaction when the prompt is carefully crafted, and support a more diverse group of learners. In this particular instance, the lesson on linguistic anthropological approaches to "performance" had tended towards dryness, and students in previous semesters frequently did not reflect an understanding of the subject matter. The use of Photovoice appears to have enhanced student learning, as well as promoted the application of course concepts to the real world context.

At first glance, the Photovoice method might appear too subjective with not enough rules, but it appears it can be quite effective in prompting students to exhibit their knowledge in a myriad of ways. As student responses and comments suggest, a well-crafted Photovoice discussion prompt can deepen student understanding of course concepts. Structuring the method is important, as it gives students the support to build knowledge, but allows them the freedom to pick the personal direction (through pictures). As one says, "I loved how the directions were clear and the time frame was spaced out." Peer interaction must play a critical role within the discussion. For instance, students depended on each other's interpretations in order to reflect on them. By its very nature, this strategy prevents "monologues” in which students are interacting with the discussion prompt, rather than each other. This freedom allows for interesting interactions among students. From the instructor's perspective, this strategy presented several advantages: first, the visual responses and student interaction generated richer, more interesting assignment submissions from students. Knowing that the discussion prompt guaranteed peer interaction alleviated the burden of facilitation from the instructor. The fact that the discussion was broken down in three parts also made grading easier because the smaller posts allowed the instructor to zero in on whether the student "got" the concept.

Findings from this exploratory study suggest that this approach is effective, but there are some lessons learned and recommendations to offer. Students suggested that the instructor should encourage them to "think outside the box" and post pictures of performances that are not so obvious. They thought that a specific example from the instructor would help with this. For this reason, it is suggested that the instructor model the discussion and offer an image that may be deemed unconventional, to encourage more risk-taking among students. Some even suggested ramping up the response from 100-200 words to 200-300, to make it more challenging. The largest barrier was students not getting a response from their peers about their images. As one student noted, "The lack of a classmate's response presented a unique challenge that I am hopeful I will not be penalized for." It may be necessary to penalize students who do not follow this instruction. The instructor found that sending out a reminder about this around the time that Part B is due was helpful in subsequent semesters. Some technical difficulties were encountered by students trying to upload their pictures. Instructions on how to upload and post the image are important to ease the technical difficulty of the task. Finally, we encourage a debriefing of the activity, allowing the class to interpret the photos together and collaboratively generate an overall analysis. In this instance, the instructor created a series of collages with student images and held a class-wide ungraded discussion.

\section{Conclusion}

This case study examines Photovoice as a potential strategy to support authentic learning within online discussions. We expect that these findings will support online instructors who are eager to 
incorporate strategies to support students' active learning and engagement. Instructors considering this technique should keep in mind that this strategy must be supported by a carefully crafted prompt and clear instructions. Connecting the Photovoice method to course concepts is key to ensuring the strategy's success. In future studies, researchers plan to collect data from more students in varied course sections, as well as analyze data beyond surveys, looking at the content contained within the discussions themselves. This will allow us to go beyond perceptions, and more accurately assess the degree of learning taking place within the online discussions. The idea of having the whole community (in this case, a classroom) interpret images will also be explored. Future research should focus on student outcomes when compared to a standard text-based discussion prompt. Another promising area of research is a multi-modal analysis of the images and text generated by the students as part of this discussion. Nevertheless, this exploratory case study suggests that this classroom strategy holds significant potential to support authentic learning in an online class setting.

While helpful, this case study presents some limitations. First, it is exploratory. Data was collected in one course taught by one professor. The sample is small, limiting the generalizability of the findings. Longitudinal data collection will further strengthen the findings of this study, as would studying applications of this strategy in other online courses over time. Importantly, this study only measured student perceptions of their own experience, but did not attempt to measure whether this perception corresponded with measurable changes in the experience of authentic learning, critical thinking, and engagement or interaction. These questions will be pursued in a future publication. Collecting future data will further support this research.

\section{Acknowledgements}

The authors would like to acknowledge the invaluable contributions of Dr. Chuck Dziuban and Dr. Patsy Moskal from the Research for Teaching Effectiveness at the University of Central Florida, who provided assistance with data analysis.

\section{Appendices}

Appendix A: Survey questions.

5-point Likert scale: Agree-somewhat agree-neither agree nor disagree-somewhat disagreedisagree

1. This discussion activity required me to use my critical thinking abilities.

2. This discussion activity challenged the way I think.

3. This discussion activity prompted me to reflect on how I think rather than what I think.

4. This discussion activity encouraged me to think about the class content in a new way.

5. This activity encouraged me to think "outside of the box."

6. Only being provided with an image (and not an interpretation) encouraged me to think more critically about the term 'performance'.

7. Interpreting others' images prompted me to think about the concept of performance in multiple ways.

8. Reading how peers interpreted my image prompted me to think about the concept of performance in multiple ways. 
9. This discussion activity allowed me to interact with the class content in a way that was unique to my personal interests.

10. This discussion activity prompted me to relate my experience of the world to the class content.

11. I found this discussion activity to be relevant to my everyday life.

12. This discussion activity made me put my knowledge of "performance" into action.

13. This discussion activity served as a learning experience in and of itself.

14. This discussion activity prompted me to create a useful product (image) to be shared with the class community.

15. This discussion activity made me think more critically about the world around me.

16. I enjoyed doing this activity.

17. I was more motivated to participate in this discussion when compared with other discussions.

18. I found this discussion activity: (Exciting; intriguing; both; neither)

19. I think this assignment is valuable.

20. This discussion activity held my attention longer than other discussion activities.

21. Interpreting the images that my peers posted made me feel more engaged in the discussion activity.

22. This activity should be used in future sections of this course.

23. This activity promoted interactions with my classmates.

24. Reading my peers' responses about my image encouraged me to reflect on my understanding of the course content.

25. Analyzing the images taken within my group encouraged me to reflect on my understanding of the course content.

26. This discussion activity strengthened the sense of community I feel in this class.

27. The instructions were clear. I knew what I was supposed to do.

28. What device did you use to take the image? (phone; tablet; camera)

29. What device did you use to add your image to the discussion? (phone; tablet; computer/laptop)

30. What statement is most accurate when thinking about the level of technical difficulty when posting your picture in the discussion? (no difficulty; some difficulty; much difficulty) 31. Please use this space to give me any additional feedback about this assignment. How could it be improved for next time?

\section{Appendix B: Compliance with Ethical Standards.}

Funding: This study did not receive funding from any source.

Ethical Approval: All procedures performed in studies involving human participants were in accordance with the ethical standards of the institutional and/or national research committee and with the 1964 Helsinki declaration and its later amendments or comparable ethical standards. This study was approved by the University of XXXX Internal Review Board (IRB) for the protection of human subjects.

Informed Consent: Informed consent was obtained from all individual participants included in the study.

\section{References}


Ahearn, L. (2012). Living Language: An introduction to linguistic anthropology. Hoboken, NJ: Wiley-Blackwell.

Behar-Horenstein, L.S., \& Niu, L. (2011). Teaching critical thinking skills in higher education: A review of the literature. Journal of College Teaching \& Learning, 8(2), 25-41.

Blow, C. (2014). “Opinion: The Self(ie) Generation.” The New York Times. March 7.

Bonnycastle, M.M., \& Bonnycastle, C.R. (2015). Photographs generate knowledge: Reflections on experiential learning in/outside the social work classroom. Journal of Teaching in Social Work, 35(3), 233-250.

Bonwell, C. C., \& Eison, J. A. (1991). Active learning: Creating excitement in the classroom. (ASHEERIC Higher Education Report No. 1). George Washington University: Washington, D.C.Chen, B., Seilhamer, R., Bennett, L., \& Bauer, S. (2015). Students' mobile learning practices in higher education: A multi-year study. EDUCAUSE Review. Retrieved on January 11, 2017, from

http://er.educause.edu/articles/2015/6/students-mobile-learning-practices-in-higher-education-amultiyear-study

Cook, K. \& Quigley, C. (2013). Connecting to our community: Utilizing photovoice as a pedagogical tool to connect college students to science. International Journal of Environmental and Science Education, 8(2), 339-357.

Dahlstrom, E. \& Bichsel, J. (2014). ECAR study of undergraduate students and information technology, 2014. Research report. Louisville, CO: ECAR, October 2014.

deNoyelles, A., Zydney, J., \& Chen, B. (2014). Strategies for creating a community of inquiry through online asynchronous discussions. MERLOT Journal of Online Teaching and Learning, 10(1), 153-165.

Edwards, M., Perry, B., Janzen, K., \& Menzies, C. (2012). Using the artistic pedagogical technology of photovoice to promote interaction in the online post-secondary classroom: The students' perspective. The Electronic Journal of e-Learning, 10(1), 32-43.

Fiorella, L., \& Mayer, R.E. (2015). Learning as a generative activity: Eight learning strategies that promote understanding. New York: Cambridge University Press.

Gubrium, A.C., \& Torres, M.I. (2013). The message in the bottle: Latino youth communicating double standard ideologies through photovoice. American Journal of Health Education, 44(3), 146-155.

Hew, K.F., Cheung, W.S., \& Ng, C.S. (2010). Student contribution in asynchronous online discussion: a review of the research and empirical exploration. Instructional Science, 38, 571606. 
Jecklin, R. (2010). Online simulation of health care reform: Helping health educators learn and participate. Global Journal of Health Education and Promotion, 13(1), 90-99.

Johnson, G. (2011). A child's right to participation: Photovoice as methodology for documenting experiences of children living in Kenyan orphanages. Visual Anthropology Review, 27(2), 141161.

Kroeger, S., \& Meyer, H. (2005). Photovoice as an educational action research tool. Paper presented at the annual meeting of the American Educational Research Association, Montreal, Canada.

LaDonna, K., \& Ravenek, M. (2014). Challenges and strategies for conducting qualitative research with persons diagnosed with rare movement disorders. The Qualitative Report, 19(16), $1-18$.

Lombardi, M.M. (2007). Authentic learning for the $21^{\text {st }}$ century: An overview. EDUCAUSE Learning Initiative Paper. Retrieved January 11, 2017 from https://net.educause.edu/ir/library/pdf/ELI3009.pdf

Mazzolini, M., \& Maddison, M. (2007). When to jump in: the role of the instructor in online discussion forums. Computers \& Education, 49(2), 193-213.

Schell, K., Ferguson, A., Hamoline, R., Shea, J., \& Thomas-Maclean, R. (2009). Photovoice as a teaching tool: Learning by doing with visual methods. International Journal of Teaching and Learning in Higher Education, 21(3), 340-352.

Yang, Y., Newby, T., \& Bill, R. (2005). Using Socratic questioning to promote critical thinking skills through asynchronous discussion forums in distance learning environments. The American Journal of Distance Education, 19(3), 163-181. 